\title{
Classical-quantum correspondence and backreaction
}

\author{
Tanmay Vachaspati ${ }^{1}$ and George Zahariade ${ }^{1,2}$ \\ ${ }^{1}$ Physics Department, Arizona State University, Tempe, Arizona 85287, USA \\ ${ }^{2}$ Beyond Center for Fundamental Concepts in Science, Arizona State University, \\ Tempe, Arizona 85287, USA
}

(Received 29 June 2018; published 5 September 2018)

\begin{abstract}
We work in the Heisenberg picture to demonstrate the classical-quantum correspondence (CQC) in which the dynamics of a quantum variable is equivalent to that of a complexified classical variable. The correspondence provides a tool for analyzing quantum backreaction problems which we illustrate by a toy model in which a rolling particle slows down due to quantum radiation. The dynamics found using the $\mathrm{CQC}$ is in excellent agreement with that found using the much more laborious full quantum analysis.
\end{abstract}

DOI: 10.1103/PhysRevD.98.065002

A large class of physical systems involve classical dynamics that is coupled to quantum degrees of freedom (d.o.f.) that get excited as the classical system evolves. Examples of such systems include particle production during cosmological evolution, Hawking radiation during gravitational collapse and Schwinger pair production in an electric field. The key question we address in this paper is: how do we account for the backreaction of the quantum excitations on the classical background? The question is of fundamental interest as its solution may hold the key to many problems of current interest including the black hole information paradox.

Past work on the backreaction question is usually framed as a perturbative-iterative process; the radiation is calculated in perturbation theory, the backreaction is then calculated semiclassically, which then leads to modified radiation, and so on. In the present work, we instead develop a classical-quantum correspondence (CQC) using which we can transform the quantum radiation problem into a classical radiation problem. Then the entire problem, including backreaction, can be cast as a set of classical equations with definite initial conditions [1,2]. These equations can then be solved numerically. (Other work on classical-quantum connections includes [3-7].)

The system we have in mind consists of a classical background variable that couples to a free quantum field. Expanding the field in modes, the mode coefficients behave like an infinite set of simple harmonic oscillators with timedependent mass and frequency. By redefining variables, it is

Published by the American Physical Society under the terms of the Creative Commons Attribution 4.0 International license. Further distribution of this work must maintain attribution to the author(s) and the published article's title, journal citation, and DOI. Funded by SCOAP . possible to eliminate the time-dependence of the mass. Thus the field theory problem can be mapped into a quantum mechanics problem consisting of an infinite set of simple harmonic oscillators with time-dependent frequencies determined by the dynamics of the background variable.

The quantum simple harmonic oscillator (qSHO) with a time-dependent frequency has been solved in terms of a two-dimensional classical SHO (cSHO) in early work [8-10] and more recently [11]. In Ref. [2], we have used this connection in the Schrödinger picture to show the equivalence of the quantum and classical systems, and we have discussed the excitations produced due to the timedependence of the frequency. This result can be applied to a mode by mode analysis of a free quantum field to show that the quantum field dynamics can be described in terms of the classical dynamics of a corresponding system with prescribed initial conditions [12]. However, particle production is usually discussed in the Heisenberg picture using the method of Bogoliubov transformations (e.g., [13]) while functional Schrödinger derivations are less familiar. In this paper we first close this gap by demonstrating the classicalquantum correspondence (CQC) in the Heisenberg picture.

The production of particles due to the time varying background will cause dissipation in the time variation. The CQC provides a simple tool to study this backreaction because quantum dynamics can be replaced by classical dynamics. To assess the validity of the CQC approach to backreaction, we construct a toy model in which we can find the backreaction using the CQC and also in the full quantum theory. The results are in excellent agreement and the accuracy of the CQC approach increases as the background becomes more classical.

This paper is organized as follows. We first show the CQC between a qSHO and two cSHOs in the Heisenberg picture when the frequency of the SHOs is an arbitrarily varying function of time. This is done in three steps. First, 
in Sec. I A we derive the Heisenberg equations of motion for the ladder operators with a time-dependent frequency. Then in Sec. I B we find the energy radiated in particles by the method of Bogoliubov transformations. In Sec. IC we show that the dynamics of the radiation and, in particular, the energy in quantum radiation, can be found by a purely classical calculation that involves doubling the radiative d.o.f., or equivalently complexifying these d.o.f. Having thus established the CQC, we turn to the quantum radiation backreaction on the dynamics of the classical variable. We find the results obtained using the CQC and compare them to the full quantum dynamics that are found by using novel, though laborious, numerical methods described in the Appendix. Our conclusions are given in Sec. III.

\section{CQC IN HEISENBERG PICTURE}

\section{A. Heisenberg equations}

The Hamiltionian for a simple harmonic oscillator with time-dependent frequency is

$$
H=\frac{p^{2}}{2 m}+\frac{m \omega^{2}}{2} x^{2}
$$

where $\omega=\omega(t)$ is an unspecified function. We define ladder operators in the usual way

$$
a=\frac{p-i m \omega x}{\sqrt{2 m \omega}}, \quad a^{\dagger}=\frac{p+i m \omega x}{\sqrt{2 m \omega}}
$$

It is straightforward to check that $\left[a, a^{\dagger}\right]=1$ even for a time-dependent $\omega$. Then,

$$
H=\omega(t)\left(a^{\dagger} a+\frac{1}{2}\right)
$$

and

$$
\frac{\partial a}{\partial t}=-\frac{\dot{\omega}}{2 \omega} a^{\dagger}, \quad \frac{\partial a^{\dagger}}{\partial t}=-\frac{\dot{\omega}}{2 \omega} a
$$

We now go to the Heisenberg picture. Then the equation of motion for $a$ is

$$
\begin{aligned}
\frac{d a}{d t} & =-i[a, H]+\frac{\partial a}{\partial t} \\
& =-i \omega a-\frac{\dot{\omega}}{2 \omega} a^{\dagger}
\end{aligned}
$$

and similarly

$$
\frac{d a^{\dagger}}{d t}=+i \omega a^{\dagger}-\frac{\dot{\omega}}{2 \omega} a
$$

\section{B. Bogoliubov transformation}

To obtain the excitation of the simple harmonic oscillator due to the time-dependence of $\omega$, we write

$a(t)=\alpha(t) a_{0}+\beta(t) a_{0}^{\dagger}, \quad a^{\dagger}(t)=\alpha^{*}(t) a_{0}^{\dagger}+\beta^{*}(t) a_{0}$

where $a_{0}$ and $a_{0}^{\dagger}$ are the annihilation and creation operators in Eq. (2) at the initial time, $t=0$. The commutation relation $\left[a, a^{\dagger}\right]=1$ leads to the constraint

$$
|\alpha|^{2}-|\beta|^{2}=1 \text {. }
$$

and Eqs. (5), (6) lead to

$$
\begin{aligned}
& \dot{\alpha}=-i \omega \alpha-\frac{\dot{\omega}}{2 \omega} \beta^{*} \\
& \dot{\beta}=-i \omega \beta-\frac{\dot{\omega}}{2 \omega} \alpha^{*}
\end{aligned}
$$

These equations also lead to the constraint

$$
\beta \dot{\alpha}-\alpha \dot{\beta}=\frac{\dot{\omega}}{2 \omega} .
$$

The expectation value of the energy in the vacuum state is

$$
E_{q}(t) \equiv\langle H\rangle=\omega(t)\left(|\beta|^{2}+\frac{1}{2}\right) .
$$

\section{The CQC}

Here we show that the quantum dynamics of the timedependent simple harmonic oscillator is given by the classical dynamics of two classical simple harmonic oscillators if we impose certain initial conditions. In contrast to the earlier derivation in the functional Schrödinger picture $[2,14,15]$, here we show this correspondence in the Heisenberg picture. Particle production is then given by the Bogoliubov transformation method, and the energy in quantum excitations, $E_{q}$, is also identical to the energy of two classical simple harmonic oscillators.

We rewrite Eqs. (9) and (10) as a single second order equation by doing the change of variables

$$
\alpha=\sqrt{\frac{m}{2 \omega}}\left(\dot{z}^{*}-i \omega z^{*}\right),
$$

$$
\beta=\sqrt{\frac{m}{2 \omega}}(\dot{z}-i \omega z),
$$

where 


$$
z \equiv \xi+i \chi
$$

is complex and $\xi$ and $\chi$ are its real and imaginary parts. The overall factor of $\sqrt{m}$ in Eqs. (13) and (14) ensures that $\alpha$ and $\beta$ have the correct mass dimensions equal to zero when $z$ has dimensions of length.

The expressions for $\alpha$ and $\beta$ are identical to the definition of the annihilation operator $a$ in Eq. (2) if we think of $z$ and $m \dot{z}$ as representing the complexified position and momentum operators for one dynamical variable and similarly $z^{*}$ and $m \dot{z}^{*}$ for a second dynamical variable. Similarly the complex conjugates $\alpha^{*}$ and $\beta^{*}$ then correspond to the expression for the creation operator $a^{\dagger}$ in Eq. (2).

Inserting Eqs. (13) and (14) in Eqs. (9) and (10) we find the equation of motion satisfied by $z$,

$$
\ddot{z}+\omega^{2}(t) z=0,
$$

which describes the dynamics of a two-dimensional cSHO (e.g., a point mass attached to a rotating spring on a plane) with time dependent frequency, or equivalently, two such one-dimensional cSHOs. Hence $\xi$ and $\chi$ (the twodimensional Cartesian coordinates of the point mass) satisfy the classical equations of motion

$$
\ddot{\xi}+\omega^{2}(t) \xi=0, \quad \ddot{\chi}+\omega^{2}(t) \chi=0 .
$$

The initial condition, $a(0)=a_{0}$, corresponds to: $\alpha(0)=1$, $\beta(0)=0$, which imply

$$
z(0)=\frac{-i}{\sqrt{2 m \omega_{0}}}, \quad \dot{z}(0)=\sqrt{\frac{\omega_{0}}{2 m}},
$$

and are equivalent to

$$
\begin{aligned}
& \xi(0)=0, \quad \dot{\xi}(0)=\sqrt{\frac{\omega_{0}}{2 m}} ; \\
& \chi(0)=\frac{-1}{\sqrt{2 m \omega_{0}}}, \quad \dot{\chi}(0)=0
\end{aligned}
$$

where $\omega_{0}=\omega(0)$. (The expressions slightly differ from those in [2] because of different conventions.) The initial conditions and Eq. (17) imply that the Wronskian is conserved,

$$
W \equiv \xi \dot{\chi}-\chi \dot{\xi}=\frac{1}{2 m}
$$

which can also be written as

$$
z^{*} p_{z}-p_{z}^{*} z=i
$$

where $p_{z} \equiv m \dot{z}$.

The initial conditions have the simple interpretation that the two-dimensional cSHO initially has the same energy as the qSHO in its ground state. This is easy to see because $\beta(0)=0$ in Eq. (12) gives $E_{q}(0)=\omega_{0} / 2$. What is more novel is that the initial conditions are such that the twodimensional cSHO also has angular momentum $L=m W=1 / 2$. Furthermore, the angular momentum, equivalently the Wronskian, is conserved during the evolution. If we think of the qSHO as the mode coefficient of a free scalar quantum field, the initial conditions imply that each mode of the corresponding classical complex scalar field must carry a conserved nonzero global charge.

The quantum dynamical problem has thus transformed into a classical evolution problem for any time-dependent frequency $\omega(t)$. To emphasize this point we write the full time-dependent annihilation operator in terms of classical solutions,

$$
a(t)=\frac{\left(p_{z}^{*}-i m \omega z^{*}\right)}{\sqrt{2 m \omega}} a_{0}+\frac{\left(p_{z}-i m \omega z\right)}{\sqrt{2 m \omega}} a_{0}^{\dagger}
$$

where $z$ denotes a classical solution with the initial conditions given above. Thus we have a mapping between the quantum solution and the classical solution.

Finally we re-express the quantum energy in Eq. (12) in terms of the $\xi$ and $\chi$ variables,

$$
\begin{aligned}
E_{q} & =\frac{\left|p_{z}\right|^{2}}{2 m}+\frac{m \omega^{2}}{2}|z|^{2} \\
& =\left(\frac{m}{2} \dot{\xi}^{2}+\frac{m \omega^{2}}{2} \xi^{2}\right)+\left(\frac{m}{2} \dot{\chi}^{2}+\frac{m \omega^{2}}{2} \chi^{2}\right) \\
& \equiv E_{\xi}+E_{\chi} .
\end{aligned}
$$

To summarize: to find the energy in quantum excitations, we simply have to solve the classical problem in Eq. (16) with the initial conditions in (18) and then calculate $E_{q}$ using (23). This is the CQC, earlier derived in the functional Schrödinger picture [2], but derived here in the Heisenberg picture via Bogoliubov transformations.

We should insist on the fact that Eq. (16) along with the initial conditions (18) is simply a rewriting of Eqs. (9) and (10) (with the associated initial conditions $\alpha(0)=1$ and $\beta(0)=0)$. Hence there is no leeway to map the quantum problem to a different classical problem, say that of only one cSHO, or two cSHOs with different initial conditions. In other words the mapping requires the existence of a conserved quantity (the angular momentum of a twodimensional cSHO) whose value is set by the constraint (8).

\section{BACKREACTION}

In the quantum problem, the time-dependent frequency produces quantum excitations and must backreact on the source responsible for the time dependence. In many situations, especially in gravitational settings, the quantum backreaction is difficult to calculate. However, the backreaction in the corresponding classical problem is in 
principle straightforward to evaluate because the classical equations of motion are known. If the classical equations are difficult to solve analytically, we can always, in principle, solve them numerically. We will now illustrate such a backreaction calculation for a toy problem that can be solved completely. This will tell us if the solution using the CQC is a good approximation to the full quantum solution.

Our toy model consists of two quantum d.o.f., $x$ and $z$, where $x$ represents a particle rolling down a linear potential and $z$ represents a simple harmonic oscillator that couples to the rolling particle. (The model has similarities to field theories used in inflationary cosmology and to the "bottomless" potentials considered in Ref. [16].) The Hamiltonian for the system is

$$
H=\frac{p_{x}^{2}}{2 M}-M a x+\frac{p_{z}^{2}}{2 m}+\frac{1}{2} m \omega_{0}^{2} z^{2}+\frac{\lambda}{2} x^{2} z^{2}
$$

which we shall rescale and write with redefined $a, \omega_{0}$ and $\lambda$ as

$$
H=\frac{p_{x}^{2}}{2}-a x+\frac{p_{z}^{2}}{2}+\frac{1}{2} \omega_{0}^{2} z^{2}+\frac{\lambda}{2} x^{2} z^{2} .
$$

Here $a$ corresponds to the constant classical acceleration while rolling, $\omega_{0}$ is the simple harmonic oscillator frequency in the absence of any coupling to the rolling particle, and $\lambda$ is the coupling.

We are mainly interested in the dynamics of the rolling particle and how the presence of the simple harmonic oscillator backreacts on the dynamics. So we will first solve the classical rolling problem, then find the simple harmonic oscillator solution in the "fixed background" approximation. Next we will solve for the full dynamics using the CQC described above. Finally we will solve the full quantum problem and compare with the result obtained using the CQC.

\section{A. Classical solution}

The classical equations of motion are

$$
\ddot{x}=a-\lambda x z^{2}, \quad \ddot{z}=-\left(\omega_{0}^{2}+\lambda x^{2}\right) z
$$

If the initial conditions (at $t=0$ ) are

$$
x(0)=0, \quad \dot{x}(0)=0, \quad z(0)=0, \quad \dot{z}(0)=0
$$

then the solution is

$$
x(t)=\frac{1}{2} a t^{2}, \quad z(t)=0 .
$$

That is, the rolling particle continues to roll with constant acceleration while the simple harmonic oscillator d.o.f. is not excited.

\section{B. Fixed background analysis}

In the fixed background of the rolling particle, the CQC is exact and the Hamiltonian for the simple harmonic oscillator is

$$
H_{z}=\frac{p_{z}^{2}}{2}+\frac{1}{2} \omega^{2}(t) z^{2}
$$

where $z=\xi+i \chi$ and

$$
\omega^{2}(t) \equiv \omega_{0}^{2}+\frac{\lambda}{4} a^{2} t^{4}
$$

Then the energy of the simple harmonic oscillator can be found from Eq. (23) where we need to solve the classical equations of motion in Eq. (17) with the initial conditions in Eq. (19). With $\omega_{0}=0$, Eq. (17) can be solved in terms of Bessel functions but for $\omega_{0} \neq 0$ we have to resort to a numerical computation. The result for $E_{q}(t)$ with $\omega_{0}=1$, $a=1, \lambda=1$ is shown in Fig. 1. Note that total energy is not conserved in the fixed background analysis: initially the energy is $\omega_{0} / 2=0.5$ while at $t=20$ we see that it has grown to $\sim 25$.

\section{Backreaction with CQC}

To obtain dynamics with backreaction with the CQC, we need to solve the classical equations

$$
\begin{gathered}
\ddot{x}=a-\lambda x\left(\xi^{2}+\chi^{2}\right), \\
\ddot{\xi}=-\left(\omega_{0}^{2}+\lambda x^{2}\right) \xi, \\
\ddot{\chi}=-\left(\omega_{0}^{2}+\lambda x^{2}\right) \chi,
\end{gathered}
$$

with initial conditions

$$
x(0)=0, \quad \dot{x}(0)=0,
$$

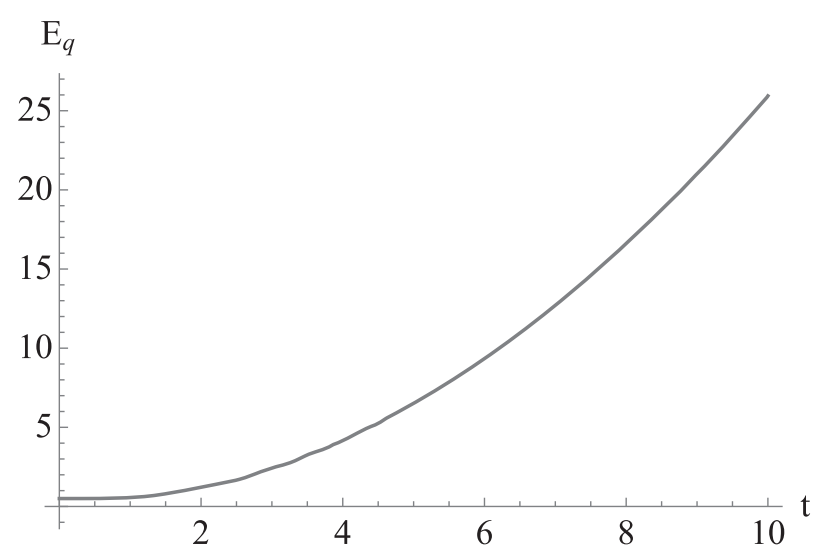

FIG. 1. $E_{q}$ vs $t$ in "fixed background approximation" for $\omega_{0}=1, a=1$. The energy of the background is conserved but the total energy is not conserved. 


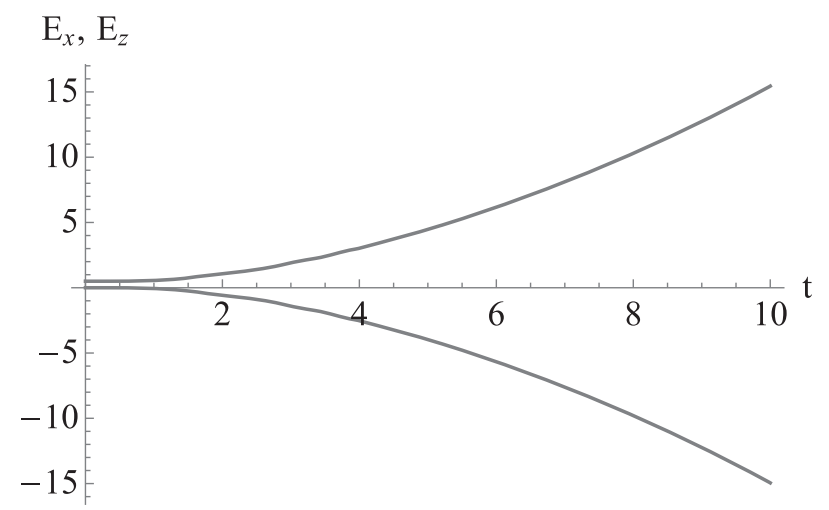

FIG. 2. The energy in the simple harmonic oscillator versus time (upper curve) as calculated with the CQC. The lower curve shows the energy in the rolling particle. The interaction term $x^{2} z^{2} / 2$ is included in the energy of the simple harmonic oscillator (upper curve). The total energy is conserved.

$$
\begin{gathered}
\xi(0)=0, \quad \dot{\xi}(0)=\sqrt{\frac{\omega_{0}}{2}}, \\
\chi(0)=\frac{-1}{\sqrt{2 \omega_{0}}}, \quad \dot{\chi}(0)=0 .
\end{gathered}
$$

This system of equations is solved numerically.

In Fig. 2 we show how the energy in the simple harmonic oscillator grows with time and that in the rolling particle decreases with time. The total energy is conserved. We will show the solution for $x(t)$ using the CQC below, after we have discussed the solution of the full quantum problem.

\section{Full quantum treatment}

To solve for the full quantum dynamics, we have to solve the time-dependent Schrödinger equation

$$
H \psi(x, z, t)=i \frac{\partial \psi}{\partial t}
$$

with $H$ given in Eq. (25). The initial wavefunction is taken to consist of Gaussian wavepackets in both the $x$ and $z$ variables,

$\psi(t=0, x, z)=\left(\frac{1}{\pi \sigma_{x}^{2}}\right)^{1 / 4} e^{-x^{2} /\left(2 \sigma_{x}^{2}\right)}\left(\frac{\omega_{0}}{\pi}\right)^{1 / 4} e^{-\omega_{0} z^{2} / 2}$

The parameter $\sigma_{x}$ is a free parameter in the full quantum problem and we shall study the dynamics for several values of $\sigma_{x}$.

With the initial condition in Eq. (38), we have $\langle x\rangle=0$ at $t=0$ for all $\sigma_{x}$. Ehrenfest's theorem in the absence of backreaction $(\lambda=0)$ gives the classical result for the evolution of the expectation value of $x$,

$$
\langle x\rangle_{\lambda=0}=\frac{1}{2} a t^{2} .
$$

We are interested in determining the effect of backreaction on this evolution.

Standard algorithms to solve the Schrödinger equation numerically, such as the explicit Crank-Nicholson method with two iterations, were found to be unstable. After some experimentation we found that Visscher's algorithm described in the Appendix is stable, provided we use a very small time step for the evolution. This limited the duration for which we could evolve the system, though it is sufficiently long to test the CQC. The numerical solution yields the wavefunction from which we then calculate the expectation value of the position of the rolling particle, $\langle x\rangle$. (Symmetry under $z \rightarrow-z$ gives $\langle z\rangle=0$ at all times.)

In Fig. 3 we show the dynamics of the rolling particle in all the different treatments: first the evolution ignoring backreaction, then the full quantum calculation for several values of $\sigma_{x}$ where backreaction is automatically included, and finally the evolution with backreaction evaluated using the CQC. It appears that the fully quantum treatment and the CQC agree to better and better accuracy as $\sigma_{x}$ grows larger. To quantify this phenomenon, we also plot the difference of quantum and CQC evolutions at a fixed time $(t=5)$ for different values of $\sigma_{x}$ (Fig. 4). The fit to the line gives

$$
\langle x\rangle_{t=5} \approx x_{c q}(t=5)+\frac{2}{\sigma_{x}} .
$$

Therefore the full quantum result goes to the CQC result in the limit of large $\sigma_{x}$.

Since the wave packet spreads during time evolution, we would also expect the agreement between CQC and fully quantum treatment to become exact at late times.

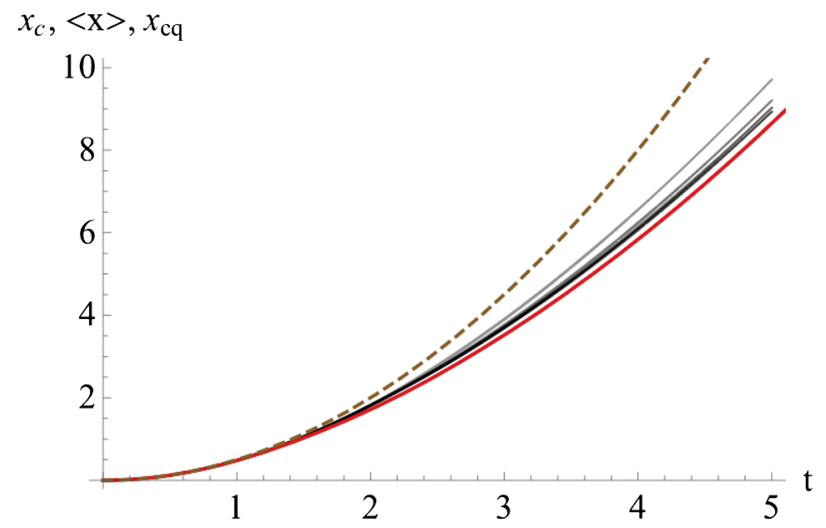

FIG. 3. Rolling as calculated in the different analyses for $a=1$, $\omega_{0}=1$ and $\lambda=1$. The dashed curve shows the classical solution, $x_{c}(t)=a t^{2} / 2$, and ignores backreaction. The gray curves show the rolling in the full quantum treatment with $\sigma_{x}=0.5,1,1.5$, 2.0, with the curves getting lower with increasing $\sigma_{x}$. The lowest (red) curve shows the rolling found using the CQC. 


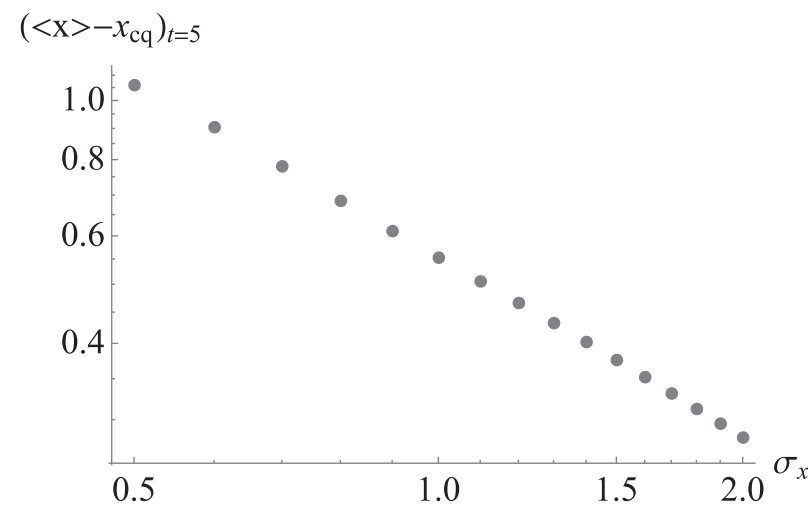

FIG. 4. Log-log plot of $\langle x\rangle-x_{c q}$ at $t=5$ showing that the CQC becomes more exact for larger $\sigma_{x}$.

To understand why this would be a reasonable expectation, we consider the time-dependent wave packet solution for a free particle,

$$
\psi(t, x)=A(t, x) \exp \left(-\frac{x^{2}}{2 \sigma_{x}^{2}} \frac{1}{1+\frac{t^{2}}{m^{2} \sigma_{x}^{4}}}\right)
$$

where $A(t, x)$ is a complex number whose modulus does not depend on $x$. Note that the parameter $\sigma_{x}$ quantifies the initial width of the wave packet and is proportional to the standard deviation on the initial position $x$ of the free particle. At late times the width of the wave packet grows as

$$
\sigma(t) \sim \frac{t}{\sigma_{x} m}
$$

So the rate of wave packet spreading is $\left(\sigma_{x} m\right)^{-1}$. This wave packet spreading is a completely quantum effect. For a rolling particle to behave classically, the rate of spreading should be much less than the rate at which it rolls,

$$
\frac{1}{\sigma_{x} m} \ll a t
$$

where $a$ is the constant acceleration of the particle. Thus a rolling particle behaves more classically at late times, and the time at which it starts behaving classically occurs earlier if the initial width of the initial wave packet is larger. Based on this behavior of a free particle, as the rolling becomes more classical, the CQC should become more exact, and at late times it should match the quantum evolution.

It is therefore instructive to study the late-time scaling behavior of the dynamics with backreaction of the rolling particle in the CQC (Fig. 5). After an early transient phase where the particle's dynamics is damped and $x(t) \propto t^{1.75}$, the acceleration asymptotically approaches a constant $\left(x(t) \propto t^{2}\right)$, albeit different from $a$. More precisely, the corresponding asymptotic solution is

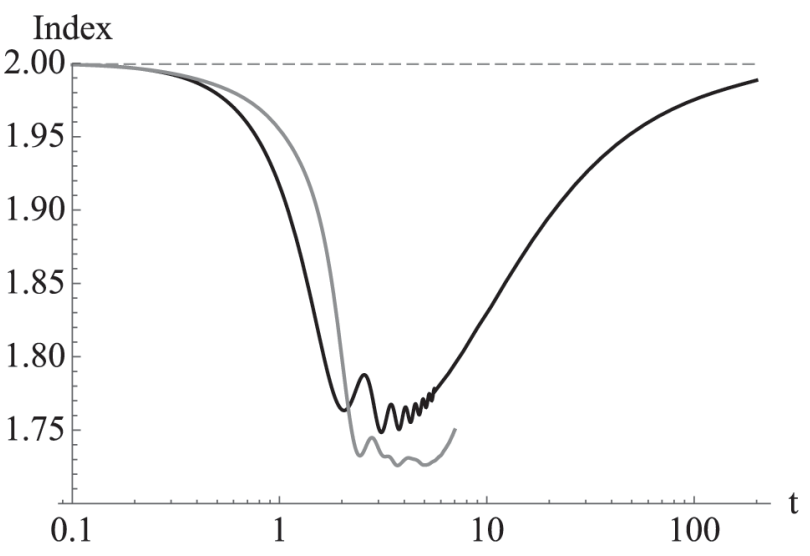

FIG. 5. Log-linear plot of the scaling index $n_{s}=d \ln (f) /$ $d \ln (t)$ for $f=x_{c q}$ (in black) and $f=\langle x\rangle_{\sigma_{x}=2}$ (in gray). At late times the scaling index of $x_{c q}$ approaches 2 .

$$
\begin{gathered}
x(t)=\frac{1}{2}\left(a-\frac{\sqrt{\lambda}}{2}\right) t^{2}, \\
\xi(t)^{2}+\chi(t)^{2}=\frac{1}{\sqrt{\lambda}\left(a-\frac{\sqrt{\lambda}}{2}\right) t^{2}} .
\end{gathered}
$$

Notice the peculiar fact that the latter equality requires the coupling to be bounded in order for this asymptotic solution to exist. (For $\lambda>4$ we find bound state solutions [16].) This renormalization of the acceleration suggests that the vacuum effectively provides a "quantum friction" force of magnitude $-\sqrt{\lambda} / 2$. In principle, this scaling behavior should also be recovered at late times in the full quantum treatment and this can be tested numerically in our toymodel. It turns out however that integrating the Schrödinger equation for longer periods of time will require a lot of computational power, or a more efficient and stable numerical algorithm. For this reason we have only plotted the scaling behavior of the fully quantum $\langle x\rangle$ (for $\sigma_{x}=2$ ) up to $t=7$ in Fig. 5 and see good agreement with the CQC. We leave a more thorough numerical analysis of the Schrödinger equation for future work.

\section{CONCLUSIONS}

We have derived the CQC in the Heisenberg picture. This shows that the dynamics of a quantum simple harmonic oscillator with a time-dependent frequency is given by the dynamics of two classical simple harmonic oscillators with the same time-dependent frequency and prescribed initial conditions. Equivalently, the quantum dynamics can be recovered by complexifying the phase space variables of the classical simple harmonic oscillator. Since the modes of a free quantum field in a background can be treated as an infinite set of simple harmonic oscillators with time-dependent frequencies, the CQC can be extended to field theory. Then the dynamics of a 
quantum real scalar field is given by the dynamics of a classical complex scalar field, again with prescribed initial conditions.

The CQC provides a tool to study the backreaction of quantum radiation on classical dynamics. We have investigated the backreaction in a toy model that involves a particle rolling down a linear potential and coupled to other simple harmonic oscillator d.o.f. We solved this toy problem using the CQC and compared it to the full quantum solution. The dynamics in the two approaches agree remarkably well, especially as the initial quantum state in the full treatment is taken to be more classical (i.e., larger $\sigma_{x}$ ). Furthermore, the analysis using the CQC is trivial to implement numerically, whereas the full quantum treatment is a nontrivial numerical task.

We can also compare the CQC with the iterative semiclassical approach to calculate the radiation and backreaction. There the radiation is calculated in a fixed background, then the background equations are solved using the radiation solution. This modifies the background and the procedure can be repeated in the modified background. In practice only a few iterations are performed and it is assumed that the procedure will converge. To assess the effectiveness of this procedure, we plot the semiclassical background $x_{N}(t)$ after $N$ iterations of this procedure (Fig. 6) as well as the fractional error when compared to the CQC result (Fig. 7). (Analogous plots can be obtained for the radiated energy.) We thus see that the iterative procedure converges quickly for $t \leq 10$, the fractional error being at the level of the working numerical precision after only 3 iterations. However as the time increases, more iterations will be needed to provide a good approximation to the full quantum problem (note that the relative error is $\sim 100 \%$ after only one iteration). The CQC is therefore superior since it bypasses this iterative semiclassical procedure and gives the exact result for all times.

We expect the CQC to have wide applicability since quantum excitations on classical backgrounds occur in

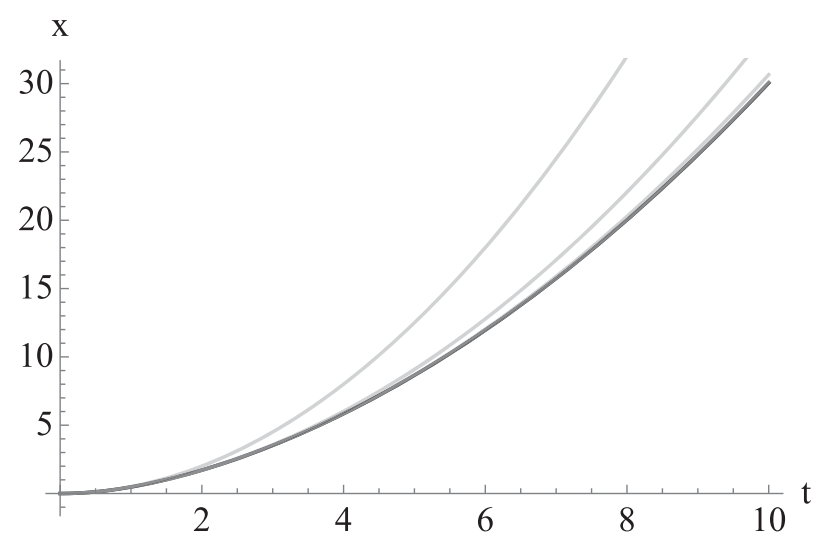

FIG. 6. Background $x_{N}$ vs $t$ for increasing number $N$ of iterations of the semiclassical procedure (gray curves) approaching the CQC result (black curve) from above.

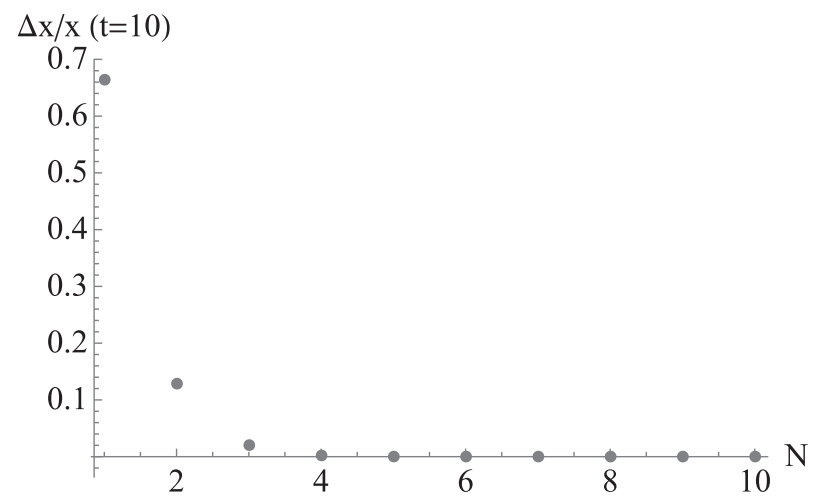

FIG. 7. Fractional error $\left(x_{N}-x_{c q}\right) / x_{c q}$ at time $t=10$ vs number of iterations $N$.

many physical systems. The approach could prove invaluable in the gravitational context where one considers quantum fields in curved spacetime. There the common approach is to work with "semiclassical gravity" [13], i.e., with the Einstein equation modified to

$$
G_{\mu \nu}=8 \pi G\left\langle T_{\mu \nu}^{\mathrm{ren}}\right\rangle
$$

where $G_{\mu \nu}$ is the Einstein tensor. The right-hand side is the expectation value of the renormalized energy-momentum tensor of the radiation fields in a suitable quantum statethis is the energy-momentum tensor after the vacuum energy has been subtracted out and other bare couplings have been adjusted to reduce the equation to the above form. In principle semiclassical gravity and extensions may provide an iterative scheme for calculating the backreaction of quantum fields on the spacetime. The CQC approach however is to solve the classical equations

$$
G_{\mu \nu}=8 \pi G T_{\mu \nu}^{\prime}
$$

where the prime on the right-hand side denotes that it is the classical energy-momentum tensor for the corresponding classical fields minus the vacuum energy contribution. (Depending on the physical situation of interest, we could include a cosmological constant term.) This modified Einstein equation would then be solved together with the classical field equations

$$
\nabla_{\nu} T^{\mu \nu \prime}=0
$$

with suitable initial conditions as discussed in this paper. The solution would provide the complete time dependence of the fields as well as the spacetime. A successful analysis in the case of gravitational collapse promises to shed light on black hole formation and the information paradox as already indicated in Ref. [2]. 


\section{ACKNOWLEDGMENTS}

We are grateful to several colleagues at the PASCOS meeting, especially Mark Hertzberg, Harsh Mathur, Paul Saffin and Andrew Tolley, for feedback. We are also grateful to Jan Olle Aguilera for a careful reading of the manuscript and to Sean Bryan for useful discussions. T. V.'s work is supported by the U.S. Department of Energy, Office of High Energy Physics, under Award No. DE-SC0013605 at Arizona State University and G. Z. is supported by John Templeton Foundation Grant No. 60253.

\section{APPENDIX: NUMERICAL METHOD}

Although our toy model Hamiltonian appears simple, standard numerical algorithms led to severe numerical instabilities. We eventually found the simple but effective algorithm due to Visscher in Ref. [17] which worked for our problem, even though we had to use very small time steps in the evolution. The idea is to write the Schrödinger equation in terms of the real and imaginary parts, $\psi_{R}$ and $\psi_{I}$, of the wavefunction

$$
\partial_{t} \psi_{R}=H \psi_{I}, \quad \partial_{t} \psi_{I}=-H \psi_{R}
$$

The novelty is that $\psi_{R}$ is taken to be at integer time steps while $\psi_{I}$ is taken to be at half-integer time steps. The equations are then discretized in the usual way by replacing spatial derivatives by central differences. The time derivative is also central which is seen e.g., by

$$
\psi_{R}(t+1, x)-\psi_{R}(t, x)=d t \times H \psi_{I}(t+1 / 2, x)
$$

As the right-hand side is evaluated half way between the times at which the differences on the left-hand side are evaluated, this gives second order accuracy in $d t$ and stability if $d t$ is small enough [17].

The probability density at any integer time step $t$ is given by

$P(t, x)=\left(\psi_{R}(t, x)\right)^{2}+\psi_{I}(t-1 / 2, x) \psi_{I}(t+1 / 2, x)$.

A good numerical check of the code is that the total probability should be unity at all times and the total energy should be conserved. Expectation values of $x$ are calculated using this expression for the probability density.

An estimate of the numerical error is obtained by evolving the Schrödinger equation forward and then backward in time. The final result should give $\langle x\rangle=0$ (the initial condition). Half the deviation gives an estimate of the numerical noise error and was negligible $\left(\sim 10^{-7}\right)$ in our check.
[1] T. Vachaspati, Phys. Rev. D 95, 125002 (2017).

[2] T. Vachaspati and G. Zahariade, arXiv:1803.08919.

[3] R. Brout, S. Massar, S. Popescu, R. Parentani, and P. Spindel, Phys. Rev. D 52, 1119 (1995).

[4] J. J. Halliwell, Phys. Rev. D 57, 2337 (1998).

[5] A. Anderson, Phys. Rev. Lett. 74, 621 (1995).

[6] F. Cooper and E. Mottola, Phys. Rev. D 40, 456 (1989).

[7] M. P. Hertzberg, J. Cosmol. Astropart. Phys. 11 (2016) 037.

[8] H. R. Lewis, J. Math. Phys. (N.Y.) 9, 1976 (1968).

[9] H. R. Lewis and W. B. Riesenfeld, J. Math. Phys. (N.Y.) 10, 1458 (1969).

[10] L. Parker, Am. J. Phys. 39, 24 (1971).

[11] K. Brown, A. Lowenstein, and H. Mathur, arXiv:1804 .01397 .
[12] T. Vachaspati and G. Zahariade, arXiv:1807.10282.

[13] N. D. Birrell and P. C. W. Davies, Quantum Fields in Curved Space, Cambridge Monographs on Mathematical Physics (Cambridge University Press, Cambridge, England, 1984), ISBN 0521278589, 9780521278584, 9780521278584, URL http://www.cambridge.org/mw/academic/subjects/physics/ theoretical-physics-and-mathematical-physics/quantum-fieldscurved-space?format=PB.

[14] T. Vachaspati, D. Stojkovic, and L. M. Krauss, Phys. Rev. D 76, 024005 (2007).

[15] M. Kolopanis and T. Vachaspati, Phys. Rev. D 87, 085041 (2013).

[16] T. Vachaspati, Phys. Rev. A 66, 014104 (2002).

[17] P. B. Visscher, Comput. Phys. 5, 596 (1991). 